\title{
Diagnosis of Iron Deficiency: Mean Corpuscular Hemoglobin (MCH) as a Predictor of Iron Deficiency in Infants
}

\author{
G. J. KNIGHT, H. DE V. HEESE, ${ }^{(16)}$ W. S. DEMPSTER, AND G. KIRSTEN \\ Department of Paediatrics and Child Health, Institute of Child Health, University of Cape Town and Red Cross War \\ Memorial Children's Hospital, Rondebosch 7700, Cape Town, Republic of South Africa
}

\begin{abstract}
Summary
Hematologic variables were measured in 240 apparently healthy infants ranging from 1-12 months of age attending a well baby clinic. There were 20 infants for each month of age. Hematologic parameters were measured in each infant by Coulter Counter Model S. Serum iron, total iron binding capacity, free erythrocyte protoporphyrin (FEP) and serum ferritin levels were measured in most infants. Their weights together with their serum iron, total iron binding capacity, and serum ferritin were judged to be independent variables of iron status, whereas the hematologic variables were considered to be response variables indicative of iron status.

The correlation coefficients among these variables, after excluding redundant variables and transforming to logarithms, were computed. Canonical correlation analysis was applied to the matrix of correlation coefficients to yield the linear function of the independent variables most highly correlated with a linear function of the response variables. The linear function of the response variables was found to be well approximated by the logarithm of the mean corpuscular haemoglobin, which was highly correlated with each of the independent variables.
\end{abstract}

\section{Abbreviations}

FEP, free erythrocyte protoporphyrin

Hb, hemoglogin

HCT, hematocrit

MCH, mean corpuscular hemoglobin

MCHC, mean corpuscular hemoglobin concentration

MCP, mean corpuscular porphyrin

MCPC, mean corpuscular porphyrin concentration

$\mathrm{MCV}$, mean corpuscular volume

RBC, red blood cell

SI, serum iron

TIBC, total iron binding capacity

TP, total porphyrin

Iron deficiency anemia is common during late infancy and in the young child (8). It is important to detect and treat iron deficiency at an early age as the systemic effects may influence the health, growth and development of infants and young children $(9,13)$.

In iron deficiency the concentration of hemoglobin is determined by the restricted supply of iron. This latter restriction may show a response in the hemoglobin $(\mathrm{Hb})$, total red blood cell count (RBC count), hematocrit (HCT), mean corpuscular volume (MCV), mean corpuscular hemoglobin (MCH), and mean corpuscular hemoglobin concentration (MCHC) (1). It may also determine the serum iron (SI), total iron binding capacity (TIBC), iron saturation \%, free erythrocyte protoporphyrin (FEP), and serum ferritin levels. There is, however, a considerable degree of overlap between normal and abnormal reference values for these tests in an infant population. Their interpretation during childhood is further compounded by factors such as age and periods of rapid growth. To overcome some of these difficulties, various combinations of tests have been suggested to assist in the diagnosis of mild iron deficiency $(3,7,10,12,13,14)$. In our hands they have not proved satisfactory and their cost prohibitive in a developing country.

A statistical exercise was embarked upon to establish the most useful single hematologic parameter for the assessment of the iron status of an infant. It formed part of a study to determine the prevalence of iron deficiency anemia during infancy in a community living in Cape Town.

\section{MATERIALS AND METHODS}

Infants. Informed consent and a full history and clinical examination of the infant was obtained in order to see whether the child fulfilled the criteria of the study. Venous blood samples were then collected. Criteria were based on a birthweight of more than $2500 \mathrm{~g}$ after a gestational period of more than $37 \mathrm{wk}$, acceptable developmental milestones and dietary habits, satisfactory gain in weight, and a normal clinical examination. Two hundred and forty apparently healthy infants ranging from 1-12 months of age were studied. There were 20 infants in each month.

The study was approved by the Ethical Review Committee of the Faculty of Medicine of the University of Cape Town. The prevalence of anemia in the community will be reported in a separate publication. The laboratory findings of this study were employed in the present statistical exercise.

Laboratory methods. $\mathrm{Hb}, \mathrm{RBC}$ count, $\mathrm{HCT}, \mathrm{MCV}, \mathrm{MCH}$ and MCHC were obtained by Coulter Counter Model S.

SI and TIBC were estimated spectrophometrically using the Ferro. Chek II Test (supplied by Hyland, Div. Travenol Laboratories Inc., Costa Weson, CA).

FEP was measured by the fluorometric assay described by Piomelli (11) and Serum Ferrintin by a two-site immunoradiometric assay (4).

Rationale for statistical approach. The iron status of an infant is the net result of iron absorption, utilization, storage, and iron excretion. It may be determined by the measurement of SI and TIBC and serum ferritin, indicative of iron absorption and storage, respectively. The infant's weight will determine its iron requirement. The values obtained from these four variables relate to one another. Their interpretation is difficult but considered jointly, they give some indication of iron status. Changes in their values result in a response in the total $\mathrm{RBC}$ count, MCV, MCHC, and FEP levels. The latter, individually or collectively, may be considered as response variables affected by the independent variables and indicative of the iron status of an individual. Hb, HCT, and $\mathrm{MCH}$ can be derived from them.

The statistical methods applied seek to find some linear combination of the four independent variables maximally correlated 
with some linear combination of the response variables as indicative of iron status in regard to its hematologic response.

Statistical methods. Canonical correlation may be viewed as a multivariate extension of multiple linear regression. In multiple linear regression only one dependent variable is permitted. In canonical correlation a number of dependent variables may be considered collectively.

The data collected were examined by this means using a computer program based on the procedure described by Cooley and Lohnes (2).

Canonical correlation is applied to a matrix of correlation coefficients arising from a set of variables measured on the same subjects. The variables are considered to belong to two subjects: the first, $x$ variables representing the left hand variables and the remaining, $y$ variables the right hand variables.

The analysis seeks to find a linear combination of the left hand variables, which yields maximum correlation, with some linear combination of the right hand variables - the first canonical correlation. The analysis then proceeds to a second and further canonical correlations in decreasing value such that the successive linear combinations of the left hand variables are uncorrelated with other linear combinations of those variables as also are the several linear combinations of the right hand variables.

The analysis is concerned with combinations of variables formed by additions and subtractions. With the data considered here many known relationships are of a multiplicative nature, e.g., the product of RBC count, MCV, and MCHC defines hemoglobin. If the logarithms of the variables replace the variables themselves linearity is ensured for many defined combinations.

In applying the procedure to the present data, the left hand variables are designated independent variables and the right hand variables as response variables.

\section{RESULTS}

One hundred and ninety-five (195) of the 240 children who entered the study had measurements for each of the four independent variables, i.e., SI, TIBC, serum ferritin, and weight, and for the response hematologic variables, i.e., RBC count, $\mathrm{MCV}$, $\mathrm{MCHC}$, and FEP. In the remaining 45 , one or more variables were missing for technical reasons.

The correlation coefficients among these variables, after transforming to logarithms, are shown in Table 1. Most of the correlation coefficients are significant $(P<0.05)$. Canonical correlation analysis applied to the matrix of correlations of Table 1 reduced the complex of Table 1 to four canonical correlations (Table 2).

The larger of these yielded a value of 0.7366 with $x^{2}=181.8337$ and 16 degrees of freedom $(P<0.001)$. The second resulted in a value of 0.3779 with $x^{2}=32.8499$ and 9 degrees of freedom, which is also considered significant $(P<0.001)$. The remaining canonical correlations resulted in $\chi^{2}$ values less than their expected values and may be ignored.

Interest then centered on the larger of the two significant canonical correlations. The corresponding coefficients, applied to the set of response variables, led to the definition of a linear function, $Y: Y=0.0717 \mathrm{y}_{1}+0.9728 \mathrm{y}_{2}+0.2199 \mathrm{y}_{3}-0.0092 \mathrm{y}_{4}(1.1)$, where $y_{1}, y_{2}, y_{3}$ and $y_{4}$ are $\log (R B C$ count), $\log (M C V), \log$
(MCHC), and $\log$ (FEP), standardized by measuring them about their respective means and dividing by their respective standard deviations. The corresponding linear function for the independent variables was found to be: $X=-0.2739 x_{1}+0.4543 x_{2}-0.4170 x_{3}$ $+0.2151 x_{4}(1.2)$ where $x_{1}, x_{2}, x_{3}$, and $x_{4}$ are $\log$ (weight), $\log (\mathrm{SI})$, $\log$ (TIBC) and $\log$ (serum ferritin), respectively. These also in standardized form. The canonical correlation between $\mathrm{X}$ and $\mathrm{Y}$ of 0.7366 (Table 2) is the greatest correlation that can be found between any linear combination of the x's and any linear combination of the y's.

A combination of the $y$ variables of the form: $X^{\prime}=a_{1} y_{1}+a_{2} y_{2}$ $+a_{3} y_{3}+a_{4} y_{4}$, where the a's are any arbitrary set of values represents a linear combination. There are clearly an infinite number of these but certain sets of "a" values define quantities which are familiar. Thus if $\mathrm{a}_{1}=\mathrm{a}_{2}=\mathrm{a}_{3}=1$ and $\mathrm{a}_{4}=0$ then $\mathrm{X}^{\prime}$ becomes formally equivalent to $\log (\mathrm{Hb})$. Various hematologic variables defined in this manner are shown in Table 3 together with the multiple correlation coefficients obtained by multiple linear regression (5) in which each of the defined hematologic variables was in turn taken as the dependent variable and regressed on the four independent variables. (A canonical correlation where there is only one right hand variable will be equal in value to the multiple correlation coefficient obtained when the right hand variable is regressed on the left hand, i.e., independent variables.)

FEP directly measured is expressed in terms of HCT. It is a measure analogous to $\mathrm{MCHC}$. It might, alternatively, be referred to as mean corpuscular porphyrin concentration (MCPC). In a similar manner (MCV)*(FEP) (ignoring a scaling factor) could be referred to as mean corpuscular porphyrin (MCP) and the product (RBC count $)^{*}(\mathrm{MCV})^{*}(\mathrm{FEP})$ referred to as total prophyrin (TP). As $(\mathrm{RBC} \text { count })^{*}(\mathrm{MCV})$ is equivalent to $10^{*}(\mathrm{HCT})$ the measure $(\mathrm{FEP}) *(\mathrm{HCT}) / 100$ is a measure of (TP), appropriately scaled. The ratio $(\mathrm{TP}) /(\mathrm{HB})$, or equivalently, $(\mathrm{FEP})^{*}(\mathrm{HCT}) /\left(100^{*}(\mathrm{HB})\right.$ has been proposed $(10,14)$ as a measure of possible utility in the investigation of iron deficiency. It is easy to show that this measure is more simply stated as (FEP)/(MCHC).

The multiple correlation coefficient for $\log (\mathrm{MCH})$ was found to be 0.7342 and therefore only marginally less than the maximum value of 0.7366 for the first canonical correlation corresponding to the undefined linear combination of 1.1. Moreover each of the determinate independent variables was found to be significant ( $P$ $<0.01)$ as a regressor.

\section{DISCUSSION}

The purpose of this paper has been to subsume into a single, coherent system a number of variables, some of which are postulated $a$ priori to be determinate of iron status and others responsive

Table 2. Estimated canonical correlations

\begin{tabular}{cccc}
\hline & Canonical correlation & Chi-square & Degrees of freedom \\
\hline 1 & 0.7366 & 181.8337 & 16 \\
2 & 0.3779 & 32.8499 & 9 \\
3 & 0.1249 & 3.5005 & 4 \\
4 & 0.0515 & 0.5067 & 1 \\
\hline
\end{tabular}

Table 1. Correlation coefficients among the variables ${ }^{1}$

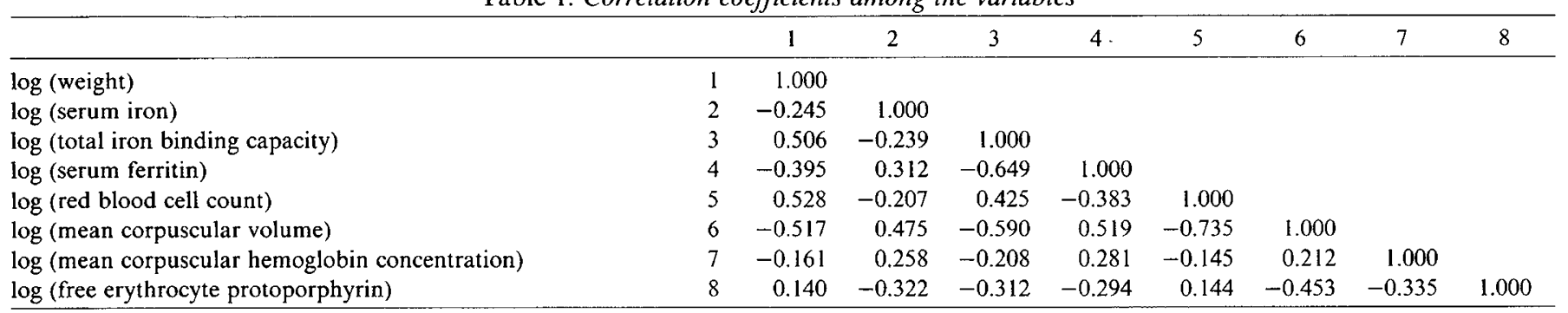

\footnotetext{
${ }^{1}$ Correlation coefficients with absolute values greater than 0.1406 are considered significant $(P<0.05)$.
} 
Table 3. Linear combinations of the indicator variables with defined equivalents

\begin{tabular}{lccccc}
\hline & $\begin{array}{c}\log \\
\text { (RBC count) }\end{array}$ & $\begin{array}{c}\log \\
\text { (MCV) }\end{array}$ & $\begin{array}{c}\log \\
\text { (MCHC) }\end{array}$ & $\begin{array}{c}\log \\
\text { (FEP) }\end{array}$ & $\begin{array}{c}\text { Multiple correlation } \\
\text { coefficient }\end{array}$ \\
\hline $\log$ (hematocrit) & 1 & 1 & 0 & 0 & 0.3897 \\
$\log$ (mean corpuscular hemoglobin) & 0 & 1 & 1 & 0 & 0.7342 \\
$\log$ (hemoglobin) & 1 & 1 & 1 & 0 & 0.4366 \\
$\log$ (mean corpuscular porphyrin) & 0 & 1 & 0 & 1 & 0.2702 \\
$\log$ (total porphyrin) & 1 & 1 & 0 & 1 & 0.3732 \\
$\log$ (TP/Hb) & 0 & 0 & -1 & 1 & 0.4287 \\
\hline
\end{tabular}

of iron status insofar as the latter are reflected in hematologic measurements.

The high first canonical correlation suggests the feasibility of expressing the interactions of independent and response variables in this way. The $\mathrm{MCH}$ was found to be the best response variable to changes in the independent variables, i.e., SI, TIBC, ferritin, and FEP. MCH is very nearly as well determined in this way as the best possible combination of the hematologic variables corresponding with the first canonical correlation. The independent variables were found to be related to $\mathrm{MCH}$ in an intuitively reasonable way in that higher values of serum iron and serum ferritin are associated with higher values of $\mathrm{MCH}$, whereas higher values of weight and TIBC act in the opposite direction. It follows that decreased values of $\mathrm{MCH}$ occur with iron deficiency.

Saarinen and Siimes (12) found lower $\mathrm{MCH}$ and MCV values in a group of infants studied compared to those who received prolonged iron supplementation, although infants with low ferritin and transferrin saturation or both had been excluded. They concluded that the differences in $\mathrm{MCH}$ and $\mathrm{MCV}$ values might indicate that these two indices are sensitive indicators of mild iron deficiency. Hershko et al. (6) also concluded that $\mathrm{MCH}$ was most suitable as an indicator of iron deficiency anemia in a rural population of children aged $1-6$ years.

The most reliable criterion of iron deficiency anemia is the hemoglobin response to a one-month therapeutic trial of iron in adequate dosage in an infant with suspected iron deficiency. Compliance in taking the medication correctly, however, is often poor and follow-up of infants difficult and expensive. For these reasons this method cannot be easily applied and alternative methods have to be explored. It is postulated that $\mathrm{MCH}$ is the best single hematologic indicator of iron status. It would be necessary to institute studies to establish the usefulness of $\mathrm{MCH}$ for the identification of infants who will respond to iron therapy.

\section{REFERENCES AND NOTES}

1. Bothwell, T. H., Charlton, R. W., Cook, J. D., and Finch. C. A.: Iron Metabolism in Man. p. 50 (Blackwell Scientific Publications, Oxford, 1979).
2. Cooley, W. W. and Lohnes, P. R.: Multivariate Procedures for the Behavioral Sciences. Chap. 3 (john Wiley and Sons, New York, 1962).

3. Dallman, P. R. and Siimes, M. A.: Percentile curves for hemoglobin and red cell volume in infancy and childhood. J. Pediatr., 94: 26 (1979).

4. Dempster, W. S., Steyn, D. L.. Knight, G. J., and Heese. H. de V.: Immunoradiometric assay of serum ferritin as a practical method of evaluating iron stores in infants and children. Med. Lab. Sci., 34: 337 (1977).

5. Efroymson, M. A.: Multiple Regression Analysis. In: R. Robston and H. Wilf. Ed.: Mathematical Methods for Digital Computers. pp. 191-203 (John Wiley and Sons, New York, 1960).

6. Herschko, C.. Bar-Or, D., Gaziel, Y., Naparstek, E., Konijn, A. M., Grossowicz, N., Kaufman, N., and Izak, G.: Diagnosis of iron deficiency anemia in a rural population of children. Relative usefulness of serum ferritin, red cell protoporphyrin, red cell indices and transferrin saturation determinations. Am. J. Clin. Nutr., 34: 1600 (1981).

7. Langer, E. E.. Haining. R. G., Labre, R. F., Jacobs, P., Crosby, E. F., and Finch, C. A.: Erythrocyte protoporphyrin. Blood, 40: 112 (1972).

8. Lanzkowsky, P.: Pediatric Hematology-Oncology. pp. 56-57. (McGraw-Hill Book Company, New York, 1980).

9. Oski, F. A.: The nonhematologic manifestations of iron deficiency Am. J. Dis. Child., 133: 315 (1979).

10. Piomelli, S., Brickman, A., and Carlos, E.: Rapid diagnosis of iron deficiency by measurement of free erythrocyte prophyrins and hemoglobin. The FEP/Hemoglobin ratio. Pediatrics, 57: 136 (1976).

11. Piomelli, S.: A micromethod for free erythrocyte porphyrins. The FEP test. J. Lab. Clin. Med., 81: 932 (1973).

12. Saarinen. M. D. and Siimes, M. A.: Developmental changes in red blood cell counts and indices of infants after exclusion of iron deficiency by laboratory criteria and continuous iron therapy. J. Pediatr., 92: 412 (1978).

13. Smith, N. J. and Rios, E.: Iron Metabolism and Iron Deficiency in Infancy and Childhood. Advances in Pediatrics, 21: 239 (Year Book Medical Publishers, Inc.. Chicago, 1974).

14. Thomas, W. J.. Koenig, H. M.. Lightsey, A. L.. and Green, R.: Free erythrocyte porphyrin: hemoglobin ratios, serum ferritin, and transferrin saturation levels during treatment of infants with iron-deficiency anemia. Blood, 49: 455 (1977).

15. We wish to thank Drs. R. J. Coogan, N. M. Durcan and M. N. Hoffman of the City of Cape Town Health Department and the staff of the Heideveld Community Health Centre for providing clinical facilities, Sister S. de Villiers for assistance, Dr. A. Bird of the Department of Hematology for control of the accuracy of the Coulter Counter Model S measurements, the South African Medical Research Council for financial assistance and Mrs. L. Makepeace for preparation of the script.

16. Requests for reprints should be addressed to: Dr. H. De V. Heese, Department of Paediatrics and Child Health. Institute of Child Health. University of Cape Town and Red Cross War Memorial Children's Hospital, Rondebosch 7700 , Cape Town, Republic of South Africa.

17. Received for publication October 2, 1981

18. Accepted for publication May 17, 1982. 